\title{
A Randomized Comparison of the Right- and Left-Sided Approaches to Ablation of the Atrioventricular Junction
}

\author{
Steven J. Kalbfleisch, MD, Brian Williamson, MD, K. Ching Man, DO, Vicken Vorperian, MD, \\ John D. Hummel, MD, Hugh Calkins, MD, S. Adam Strickberger, MD, \\ Jonathan J. Langberg, MD, and Fred Morady, MD
}

Radlofrequency ablation of the atrioventricular (AV) junction may be performed using either a right- or left-sided approach. This study prospectively compared the left-sided approach with persistent attempts from the right slde in patients in whom InItial radiofrequency applications on the right side were unsuccessful. Twenty-one of 54 patients did not have complete AV block induced after 3 right-sided radiofrequency applications. These 21 patients were randomly assigned to un dergo either the left-sided approach $(n=10)$ or to undergo additional attempts from the right side ( $n=11$ ). The right-sided approach was performed by positioning the ablation catheter to record the largest possible atrial and His bundle electrograms. The left-sided approach was performed by positioning the ablation catheter along the left ventricular septum, where a His bundle potential was recorded. If either approach was not successful after an additional 17 radiofrequency applications, the altemative approach was then used. The AV junction was successfully ablated in all 10 patients randomized to the left-sided approach, but in only 6 of 11 patients randomized to persistent right-sided attempts ( $p<0.05$ ). The 5 patients in whom the AV junction was not successfully ablated using the right-sided approach underwent the left-sided approach and had a successful outcome after a mean of $1.2 \pm 0.4$ radiofrequency applications. The left-sided approach required significantly fower radiofrequency applications after randomization than the right-sided approach ( $3 \pm 3.4$ vs $11 \pm 7.6, p<0.01$ ). In pationts in whom initial attempts at ablation of the AV junetion using a right-sided approach are unsuccessful, the left-sided approach is more effective and efficient than persistent attempts using the rightsided approach.

(Am J Cardiol 1993;72:1406-1410)

From the Department of Internal Medicine, Division of Cardiology, The University of Michigan Medical Center, Ann Arbor, Michigan. Manuscript received June 11, 1993; revised manuscript received and accepted July 2, 1993.

Address for reprints: Fred Morady, MD, University of Michigan Medical Center, 1500 East Medical Center Drive, Division of Cardiology UHB1F245, Ann Arbor, Michigan 48109-0022.
A lthough the conventional right-sided approach for radiofrequency ablation of the atrioventricular (AV) junction has a high success rate, some patients require multiple applications of radiofrequency energy to achieve a successful outcome..$^{1-6}$ In contrast, in most patients who have undergone ablation of the $\mathrm{AV}$ junction from the left ventricle, complete AV block has been induced by $<5$ applications of radiofrequency energy. ${ }^{5-7}$ Therefore, the efficiency of AV junction ablation could be improved by using the left-sided approach if initial attempts with the conventional right-sided approach are unsuccessful. However, no previous studies have prospectively compared these 2 techniques. Therefore, the present study was designed to compare, in a prospective, randomized fashion, the right- and leftsided approaches to ablation of the $\mathrm{AV}$ junction in patients in whom an initial 3 radiofrequency energy applications using the right-sided approach are ineffective.

\section{METHODS}

Study design: Fifty-four consecutive patients with symptomatic, drug-refractory, supraventricular tachycardia underwent an attempt at radiofrequency catheter ablation of the $\mathrm{AV}$ junction using the conventional rightsided approach at the University of Michigan; in 33 of these patients $(61 \%)$, complete AV block was induced by 1 to 3 applications of radiofrequency energy. The subjects of this study were the 21 patients in whom 3 applications of radiofrequency energy using the rightsided approach were unsuccessful in ablating AV conduction. Ten patients were randomly assigned to undergo the left-sided approach and 11 were randomly assigned to undergo additional attempts using the rightsided approach. If complete AV block was not achieved after 17 applications of radiofrequency energy using the technique to which the patient was randomly assigned, the alternate technique was then used.

Characteristics of subjects: The characteristics of the patients assigned to undergo the right- and left-sided approaches are described in Table I. All of the patients had severe symptoms caused by either atrial fibrillation or atrial tachycardia with an uncontrolled ventricular rate. The only difference in the clinical characteristics between the 2 groups was that the indication for AV junction ablation was paroxysmal atrial fibrillation more often in the patients assigned to the leftsided approach $(\mathrm{p}=0.04$, Table I).

Study protocol: The study protocol was approved by the Human Research Committee at the University of 
Michigan, and informed, written consent was obtained from all patients. A 6 or $7 \mathrm{Fr}$ electrode catheter was inserted into a femoral vein and positioned in the right ventricular apex for use as a temporary pacemaker. The catheter used for ablation was a 7Fr bipolar or quadripolar electrode catheter with a $4 \mathrm{~mm}$ distal electrode, 2 to $5 \mathrm{~mm}$ interelectrode spacing, and a deflectable tip (Mansfield Scientific, Boston, Massachusetts). Bipolar recordings from the distal pair of electrodes of the ablation catheter were filtered at 50 to $500 \mathrm{~Hz}$ and recorded at a gain of $80 \mathrm{~mm} / \mathrm{mV}$. The intracardiac electrograms and leads $V_{1}$, I, II and III were displayed on an oscilloscope and recorded on a Siemens-Elema Mingograph-7 recorder at a paper speed of 25 to $100 \mathrm{~mm} / \mathrm{s}$. All patients received 3,000 $\mathrm{U}$ of intravenous heparin at the start of the procedure.

Ablation protocol: The right-sided approach to ablation of the $\mathrm{AV}$ junction was performed by inserting the ablation catheter in the right femoral vein and positioning it at the anterior/superior aspect of the tricuspid annulus. Radiofrequency energy was applied at sites where the largest possible atrial and His bundle deflections were recorded.

The technique used to ablate the AV junction from the left ventricle has been described previously. ${ }^{7}$ The ablation catheter was inserted into a femoral artery and positioned in the left ventricle along the anterior septum. Radiofrequency energy was applied at sites at which the largest possible His bundle deflection was recorded, irrespective of the size of the atrial electrogram. Radiofrequency energy was delivered as an unmodulated sine wave at a frequency of $500 \mathrm{kHz}$ (EP Technologies, Inc, Mountainview, California) between the distal electrode of the ablation catheter and a large skin electrode (Valleylab, Boulder, Colorado) positioned on the posterior chest. All applications of radiofrequency energy were at an output of $35 \mathrm{~W}$ and were 30 seconds in duration.

The procedure duration and the duration of fluoroscopy from the point of randomization, including the time required for arterial cannulation in patients randomized to the left-sided approach, and the number of radiofrequency energy applications required after randomization were recorded in each case. The time at which complete $\mathrm{AV}$ block was induced was taken as the end of the ablation procedure.

The intrinsic escape rhythm 15 minutes after ablation was recorded in each patient by adjusting the temporary pacemaker to a rate of 30 beats/min for up to $2 \mathrm{~min}$ utes. If an escape rhythm emerged, its cycle length was measured.

Poatablation protocol: Patients were observed for 15 to 30 minutes after ablation of the $\mathrm{AV}$ junction. If complete $\mathrm{AV}$ block persisted, a rate-responsive ventricular pacemaker was implanted. To avoid postablation ventricular tachycardia, pacing was performed at a rate of 80 to 90 beats/min for 24 to 48 hours after the procedure. Patients underwent continuous, telemetric electrocardiographic monitoring for 2 to 3 days and were then discharged from the hospital. They were seen at followup 1 to 2 weeks and 3 months after ablation to evaluate the efficacy of the procedure and to determine the rate of the intrinsic escape rhythm. All patients were in-

\begin{tabular}{|c|c|c|}
\hline & $\begin{array}{l}\text { Right-Sided } \\
(n=11)\end{array}$ & $\begin{array}{l}\text { Left-Sided } \\
(n=10)\end{array}$ \\
\hline Age (years, mean \pm SD) & $64 \pm 10$ & $63 \pm 9$ \\
\hline Women:men & $5: 6$ & $6: 4$ \\
\hline Heart disease present & $4(36 \%)$ & $4(40 \%)$ \\
\hline \multicolumn{3}{|l|}{ Heart disease type } \\
\hline Coronary artery disease & 3 & 2 \\
\hline Hypertensive & 1 & 2 \\
\hline LVEF (mean \pm SD) & $0.51 \pm 0.09$ & $0.50 \pm 0.13$ \\
\hline Symptom duration (years) & $7 \pm 4$ & $9 \pm 5$ \\
\hline \multicolumn{3}{|l|}{ Indication for ablation } \\
\hline Paroxsysmal AF & 4 & 9 \\
\hline Chronic AF & 5 & 1 \\
\hline Atrial tachycardia & 2 & 0 \\
\hline \multicolumn{3}{|l|}{ Rhythm during ablation } \\
\hline Sinus rhythm & 5 & 7 \\
\hline Atrial fibrillation & 6 & 3 \\
\hline Number of prior medications & $4.5 \pm 3$ & $4.4 \pm 2.1$ \\
\hline
\end{tabular}

structed to contact one of the authors in the event of a recurrence of arrhythmia-related symptoms.

Statistical analysis: Values are expressed as mean \pm 1 SD. Continuous variables were analyzed using Student's unpaired $t$ test. Categorical variables were analyzed by Fishers exact test or contingency table analysis. A p value $<0.05$ was considered significant.

\section{RESULTS}

Efficacy of ablation attempts (Table II): Each of the 10 patients randomly assigned to the left-sided approach had a successful outcome, compared with 6 of the 11 patients (54\%) randomly assigned to continue with the right-sided approach $(\mathrm{p}<0.05)$. Based on an intentionto-treat analysis, the 10 patients randomized to undergo the left-sided approach required significantly fewer radiofrequency applications after randomization than did the 11 patients randomized to the right-sided approach ( $3 \pm 3.4$ vs $11 \pm 7.6, p<0.01$ ).

The 5 patients in whom the right-sided approach was ineffective in creating AV block underwent the left-sided approach and had a successful outcome after a mean of $1.2 \pm 0.4$ radiofrequency applications. Among the patients randomly assigned to the right-sided approach, there were no significant differences between those in whom the right-sided approach was successful and those who crossed over to the left ventricular approach with respect to age, gender, underlying heart disease, left ventricular ejection fraction, or rhythm at the time of the ablation procedure.

Duration of procedures: Based on an intention-totreat analysis, there were no significant differences between the patients randomly assigned to undergo the left-sided approach and those randomly assigned to the right-sided approach with respect to the time required to achieve complete AV block (18.2 \pm 12.4 vs $26.8 \pm 16.8$ minutes, $\mathrm{p}=0.2)$ or the duration of fluoroscopy $(8 \pm 8.3$ vs $16.8 \pm 13.5$ minutes, $\mathrm{p}=0.1$ ) after randomization.

Electrogtram characteristics at ablation sttes: $\mathrm{Be}-$ cause only 6 successful right-sided target sites were 


\begin{tabular}{|c|c|c|c|c|c|c|c|c|c|}
\hline Patient & $\begin{array}{l}\text { Heart } \\
\text { Disease }\end{array}$ & LVEF & $\begin{array}{c}\text { Initial } \\
\text { Approach }\end{array}$ & $\begin{array}{l}\text { Number of RF } \\
\text { Applications }\end{array}$ & Outcome & $\begin{array}{c}\text { Number of RF Appl. } \\
\text { After Crossover }\end{array}$ & $\begin{array}{l}\text { Outcome After } \\
\text { Crossover }\end{array}$ & $\begin{array}{l}\text { Follow-Up } \\
\text { (mos) }\end{array}$ & $\begin{array}{l}\text { Long-Term } \\
\text { Comp. }\end{array}$ \\
\hline 1 & SH & 0.55 & Left & 12 & $3^{\circ} \mathrm{AVB}$ & - & - & 17 & 0 \\
\hline 2 & 0 & 0.55 & Left & 1 & $3^{\circ} \mathrm{AVB}$ & - & - & 16 & 0 \\
\hline 3 & SH & 0.50 & Left & 1 & $3^{\circ} \mathrm{AVB}$ & - & - & 16 & 0 \\
\hline 4 & 0 & 0.50 & Left & 1 & $3^{\circ} \mathrm{AVB}$ & - & - & 15 & 0 \\
\hline 5 & 0 & 0.59 & Left & 3 & $3^{\circ} \mathrm{AVB}$ & - & - & 12 & 0 \\
\hline 6 & CAD & 0.54 & Left & 1 & $3^{\circ} \mathrm{A} \vee B$ & - & - & 12 & 0 \\
\hline 7 & 0 & 0.52 & Left & 4 & $3^{\circ} \mathrm{AVB}$ & - & - & 9 & 0 \\
\hline 8 & 0 & 0.55 & Left & 1 & $3^{\circ} A V B$ & - & - & 8 & 0 \\
\hline 9 & CAD & 0.15 & Left & 4 & $3^{\circ} \mathrm{AVB}$ & - & - & 3 & SD \\
\hline 10 & 0 & 0.50 & Left & 2 & $3^{\circ} \mathrm{AVB}$ & - & - & 7 & 0 \\
\hline 11 & 0 & 0.65 & Right & 17 & No AVB & 1 & $3^{\circ} \mathrm{AVB}$ & 16 & 0 \\
\hline 12 & CAD & 0.35 & Right & 17 & No AVB & 2 & $3^{\circ}$ AVB & 1 & SD \\
\hline 13 & SH & 0.45 & Right & 17 & No AVB & 1 & $3^{\circ} \mathrm{AVB}$ & 7 & 0 \\
\hline 14 & 0 & 0.55 & Right & 17 & No AVB & 1 & $3^{\circ} \mathrm{AVB}$ & 7 & 0 \\
\hline 15 & $C A D$ & 0.42 & Right & 17 & No AVB & 1 & $3^{\circ} \mathrm{AVB}$ & 14 & 0 \\
\hline 16 & CAD & 0.45 & Right & 2 & $3^{\circ} \mathrm{AVB}$ & - & - & 7 & 0 \\
\hline 17 & 0 & 0.50 & Right & 14 & $3^{\circ}$ AVB & - & - & 14 & 0 \\
\hline 18 & 0 & 0.55 & Right & 12 & $3^{\circ} \mathrm{AVB}$ & - & - & 10 & 0 \\
\hline 19 & 0 & 0.57 & Right & 1 & $3^{\circ} \mathrm{AVB}$ & - & - & 14 & 0 \\
\hline 20 & 0 & 0.53 & Right & $i$ & $3^{\circ}$ AVB & - & - & 9 & 0 \\
\hline 21 & 0 & 0.60 & Right & 6 & $3^{\circ}$ AVB & - & - & 8 & 0 \\
\hline
\end{tabular}

\begin{tabular}{|c|c|c|c|c|}
\hline & \multicolumn{2}{|c|}{ Right-Sided Sites } & \multicolumn{2}{|c|}{ Left-Sided Sites } \\
\hline & $\begin{array}{l}\text { Successful } \\
(n=39)\end{array}$ & $\begin{array}{l}\text { Unsuccessful } \\
(n=202)\end{array}$ & $\begin{array}{l}\text { Successful } \\
(n=15)\end{array}$ & $\begin{array}{l}\text { Unsuccessful } \\
(n=21)\end{array}$ \\
\hline $\begin{array}{l}\text { Atrial amplitude (mv) } \\
\text { His amplitude (mv) } \\
\text { Ventricular amplitude (mv) } \\
\text { Atrial/ventricular ratio } \\
\text { His/ventricular ratio }\end{array}$ & $\begin{array}{l}0.43 \pm 0.38 \ddagger \\
0.14 \pm 0.08^{\star} \\
0.73 \pm 0.35^{\star} \\
0.83 \pm 1.14 \ddagger \\
0.24 \pm 0.23^{\star}\end{array}$ & $\begin{array}{l}0.19 \pm 0.25 \\
0.07 \pm 0.08 \\
0.59 \pm 0.32 \\
0.56 \pm 1.23 \\
0.13 \pm 0.16\end{array}$ & $\begin{array}{l}0.13 \pm 0.16 \\
0.13 \pm 0.09 \dagger \\
0.83 \pm 0.27 \\
0.18 \pm 0.20 \\
0.19 \pm 0.19 \dagger\end{array}$ & $\begin{array}{l}0.22 \pm 0.14 \\
0.07 \pm 0.08 \\
0.86 \pm 0.27 \\
0.29 \pm 0.21 \\
0.08 \pm 07\end{array}$ \\
\hline $\begin{array}{l}{ }^{*} p<0.05 \text { versus unsuccessful } \\
t p<0.05 \text { versus unsuccessful } \\
\neq \mathrm{p}<0.05 \text { versus successful le }\end{array}$ & $\begin{array}{l}\text {-sided sttes. } \\
\text { ided sites. } \\
\text { zd sites. }\end{array}$ & & & \\
\hline
\end{tabular}

available for analysis among the 11 patients randomly assigned to the right-sided approach, the target sites among the 33 patients who had a successful outcome with initial attempts using the right-sided approach and who did not qualify for randomization were also analyzed. The electrogram characteristics of the successful and unsuccessful right- and left-sided target sites are described in Table III. Successful right-sided sites had atrial, His bundle and ventricular electrograms that were significantly larger in amplitude than the corresponding electrograms at unsuccessful right-sided sites. In comparing the successful and unsuccessful left-sided target sites, only the amplitude of the His bundle electrogram was significantly larger at successful sites than at unsuccessful sites. In comparing the successful right- and left-sided sites, the atrial electrogram amplitude and the atrial:ventricular electrogram ratio were significantly larger on the right side.

Intringle escape mythma: Fifteen minutes after ablation of AV conduction, 9 of 15 patients (60\%) who had a successful outcome with the left-sided approach had an intrinsic escape rate $<30$ beats $/ \mathrm{min}$, compared with 4 of 6 patients $(67 \%)$ who had a successful outcome with the right-sided approach $(p=0.8)$. Among patients in whom an escape rhythm was present $15 \mathrm{~min}$ utes after ablation, the mean cycle lengths of the escape rhythms in the patients who underwent the left- and right-sided approaches did not differ significantly $(1,737$ \pm 431 vs $1,500 \pm 354 \mathrm{~ms}, \mathrm{p}=0.5$ ).

Three months after the ablation procedure, 3 of 15 patients (20\%) who had a successful outcome with the left-sided approach had an intrinsic escape rate $<30$ beats/min, compared with 2 of 6 patients (33\%) who had a successful outcome with the right-sided approach $(p=0.8)$. Among patients in whom an escape rhythm was present 3 months after ablation, the mean cycle lengths of the escape rhythms in the patients who underwent the left- and right-sided approaches did not differ significantly $(1,436 \pm 189$ vs $1,560 \pm 81 \mathrm{~ms}, \mathrm{p}=0.3)$.

Longterm follow-up (Table II): During a mean of $10.6 \pm 4.5$ months of follow-up, all patients continued to have complete AV block. Two patients died sudden- 
ly during follow-up (Table II). One patient who was assigned to the left-sided approach had an ischemic cardiomyopathy and a left ventricular ejection fraction of 0.15 and died in his sleep approximately 3 months after the ablation procedure. A second patient, who was assigned to the right-sided approach but crossed over to the left-sided approach and had coronary artery disease, a history of an anterior wall myocardial infarction, and a left ventricular ejection fraction of 0.35 , had a fatal out-of-hospital cardiac arrest with documented ventricular fibrillation 1 month after the ablation procedure. A postmortem examination was not performed in either patient.

\section{DISCUSSION}

Main findings: The results of this study demonstrate that when AV block is not easily achieved with $\leq 3$ applications of radiofrequency energy using the conventional right-sided approach for catheter ablation of the $\mathrm{AV}$ junction, it may be preferable to then use the leftsided approach instead of persisting with right-sided attempts. The left-sided approach was uniformly effective in creating complete AV block, whereas persistent rightsided attempts eventually were successful in only 54\% of the patients assigned to this approach. A noteworthy indication of the value of the left-sided approach is that complete AV block was achieved by only 1 or 2 radiofrequency applications in the left ventricle, after a total of 20 applications had been ineffective in 5 patients who had been assigned to the right-sided approach.

Another indication of the superiority of the left-sided approach over continued use of the right-sided approach is that based on an intention-to-treat analysis, a mean of 11 radiofrequency applications was required to achieve success in patients randomized to the right-sided approach, compared with a mean of only 3 applications with the left-sided approach. Therefore, when radiofrequency ablation of the AV junction is not effective after 3 applications of radiofrequency energy at the tricuspid annulus, switching to the left-sided approach is more efficacious and efficient than continuing to attempt ablation from the right side.

Duration of procedures: Although fewer applications of radiofrequency energy were required to achieve complete AV block with the left-sided than with the right-sided approach, this greater efficiency was not associated with a decrease in the total duration of the ablation procedure. When switching to the left ventricular approach, several minutes are required to cannulate the femoral artery and to position the ablation catheter in the left ventricle, and this may partially offset the advantage gained by achieving a successful outcome with fewer applications of radiofrequency energy.

Comparison with previous studles: In previous reports on the efficacy of the left-sided approach to ablation of the AV junction, patients were limited to those in whom multiple applications of radiofrequency energy along the tricuspid annulus had been ineffective..$^{5-7}$ In accord with the results of the present study, complete AV block was achieved with a small number of energy applications in the left ventricle after multiple energy applications on the right side had failed. The present study is the first to demonstrate an advantage of the left ventricular approach even in patients in whom only 3 applications of radiofrequency energy on the right side have failed.

Electrogram characteristics at ablation sittes: The amplitude of the His bundle electrogram was associated with a successful outcome at both right- and left-sided target sites. However, the amplitude of the atrial electrogram was associated with successful ablation only on the right side. The mean amplitude of the atrial electrogram at effective target sites in the left ventricle was significantly smaller than at effective target sites on the right side, and no different from the mean amplitude of the atrial electrogram at ineffective left-sided sites. These results are in agreement with the results of prior studies and imply that the left ventricular approach results in ablation of a more distal portion of the AV junction. 1,2,6,7

Intrinsic escape hythms: No differences were noted either at 15 minutes or at 3 months after ablation between the right- and left-sided approaches in the proportion of patients having an escape rhythm of $<30$ beats/min, or in the mean rate of the escape rhythms faster than 30 beats $/ \mathrm{min}$. Therefore, with respect to intrinsic escape rhythms, there is no evidence of any advantage or disadvantage to the left-sided approach compared with persistent use of the right-sided approach when initial attempts with the right-sided approach are ineffective.

Complications of ablation: Two patients in this study died suddenly within 3 months after undergoing ablation of the AV junction. A successful outcome had been achieved using the left-sided approach in both patients, 1 after 3 unsuccessful right-sided radiofrequency applications, and the other after 20. Sudden death has been reported to occur in approximately $2 \%$ of patients who undergo direct-current ablation of the $\mathrm{AV}$ junction, ${ }^{8}$ but has never been reported after radiofrequency ablation. ${ }^{1-7,9}$ As was the case in most patients who died suddenly after direct-current ablation of the AV junction, the 2 patients in this study who died suddenly had severe preexisting left ventricular dysfunction. It is unclear whether their sudden deaths were related to the underlying heart disease, the ablation procedure, or a combination of both factors. However, the fact that no sudden deaths have been reported in a large number of patients who have undergone radiofrequency ablation of left- or right-sided accessory pathways or $\mathrm{AV}$ nodal reentrant tachycardia suggests that the sudden deaths in the present study are more likely to have been a complication of the underlying heart disease than the ablation procedure. ${ }^{10-18}$

Study IImitations: A major limitation of the design of this study is that it does not allow a comparison of the complication rates of the right- and left-sided approaches to radiofrequency ablation of the $\mathrm{AV}$ junction. Each patient in this study in whom radiofrequency energy was delivered in the left ventricle already had received either 3 or 20 applications of radiofrequency energy on the right side. Therefore, although all of the complications that occurred in this study occurred in patients in whom AV block was achieved using the left ventricular approach, it cannot be known whether these 
complications were attributable to the right-sided or left ventricular energy applications. A study design in which patients were randomly assigned to the right-sided and left ventricular approaches without any previous ablation attempt would have permitted a comparison of complication rates. However, because it is unlikely in clinical practice that the left ventricular approach ever would be used without first trying the right-sided approach, it was believed that this type of study design would not have been clinically relevant.

Conclusions: In patients who are appropriate candidates for radiofrequency ablation of the $\mathrm{AV}$ junction, complete AV block can be achieved easily and quickly in almost all patients using the conventional, right-sided approach to ablation. However, if AV conduction persists after 3 radiofrequency applications at the tricuspid annulus, it is more efficient to switch to the left-sided approach than to persist with the right-sided approach. In patients in whom left ventricular catheterization would be problematic, persistent attempts with the rightsided approach may be appropriate. These would include patients with aortic stenosis, a prosthetic aortic or mitral valve, and patients with occlusive arterial disease.

1. Langberg JJ, Chin MC, Rosenqvist M, Cockrell J, Dullet N, Van Hare G, Griffin JC, Scheinman MM. Catheter ablation of the atrioventricular junction with radiofrequency energy. Circulation 1989;80:1527-1535.

2. Langberg JJ, Chin M, Schamp DJ, Lee MA, Goldberger J, Pederson DN, Oeff M, Lesh MD, Griffin JC, Scheinman MM. Ablation of the atrioventricular junction with radiofrequency energy using a new electrode catheter. Am J Cardiol 1991;67: 142-147.

3. Jackman WM, Wang X, Friday KJ, Fitzgerald DM, Roman C, Moulton K, Margolis PD, Bowman AJ, Kuck KH, Naccarelli GV, Pitha JV, Dyer J, Lazzara R. Catheter ablation of atrioventricular junction using radiofrequency current in 17 patients; comparison of standard and large-tip catheter electrodes. Circulation 1991; 83:1562-1576.

4. Yeung-Lai-Wah JA, Alison JF, Lonergan L, Leather R, Kerr CR. High success rate of atrioventricular node ablation with radiofrequency energy. $J$ Am Coll $\mathrm{Car}$ diol 1991;18:1753-1758.

5. Souza O, Gursoy S, Simonis F, Steurer G, Andries E, Brugada P. Right-sided versus left-sided radiofrequency ablation of the His bundle. PACE 1992;15:
1454-1459.

6. Trohman RG, Simmons TW, Moore SL, Firstenberg MS, Williams D, Maloney JD. Catheter ablation of the atrioventricular junction using radiofrequency energy and a bilateral cardiac approach. Am J Cardiol 1992;70:1438-1443.

7. Sousa J, El-Atassi R, Rosenheck S, Calkins H, Langberg JJ, Morady F. Radiofrequency catheter ablation of the atrioventricular junction from the left ventricle. Circulation 1991;84:567-571.

8. Evans GT, Scheinman MM, Executive Committee of the Registry. The percutaneous cardiac mapping and ablation registry: final summary results. PACE 1988 1621-1626.

9. Morady F, Calkins H, Langberg JJ, Armstrong WF, deBuitleir M, El-Atassi R, Kalbfleisch SJ. A prospective randomized comparison of direct current and radiofrequency ablation of the atrioventricular junction. $J$ Am Coll Cardiol 1993;21 102-109.

10. Jackman WM, Wang X, Friday KJ, Roman CA, Moulton KP, Beckman KJ, McClelland JH, Twidale N, Hazlitt HA, Prior MI, Margolis PD, Calame JD, Overholt ED, Lazzara R. Catheter ablation of accessory atrioventricular pathways (WolffParkinson-White syndrome) by radiofrequency current. $N$ Engl J Med 1991;324 1605-1611.

11. Calkins H. Sousa J, El-Atassi R, Rosenheck S, de Buitleir M, Kou WH, Kadish $\mathrm{AH}$, Langberg JJ, Morady F. Diagnosis and cure of the Wolff-Parkinson-White syndrome or paroxysmal supraventricular tachycardias during a single electrophysiologic test. $N$ Engl J Med 1991;324:1612-1628.

12. Schluter M, Geiger M, Siebels J, Duckeck W, Kuck KH. Catheter ablation using radiofrequency current to cure symptomatic patients with tachyarrhythmias related to an accessury atrioventricular pathway. Circulation 1991;84:1644-1661.

13. Calkins H, Langberg JJ, Sousa J, El-Atassi R, Leon A, Kou WH, Kalbfleisch SJ, Morady F. Radiofrequency catheter ablation of accessory atrioventricular connections in 250 patients: abbreviated therapeutic approach to Wolff-Parkinson-White syndrome. Circulation 1992;85:1337-1346.

14. Lesh MD, Van Hare GF, Schamp DJ, Chien W, Lee MA, Griffin JC, Langberg JJ, Cohen TJ, Lurie KG, Scheinman MM. Curative percutaneous catheter ablation using radiofrequency energy for accessory pathways in all locations: results in 100 consecutive patients. $J$ Am Coll Cardiol 1992;19:1303-1309.

15. Jazayeri MR, Hempe SL, Sra JS, Dhala AA, Blanck Z, Deshpande SS, Avitall B, Krum DP, Gilbert CJ, Akhtar M. Selective transcatheter ablation of the fas and slow pathways using radiofrequency energy in patients with atrioventricular nodal reentrant tachycardia. Circulation 1992;85:1318-1328.

16. Kay GN, Epstein AE, Dailey SM, Plumb VJ. Selective radiofrequency ablation of the slow pathway for the treatment of atrioventricular nodal reentrant tachycardia: evidence for involvement of perinodal myocardium within the reentrant circuit. Circulation 1992;85:1675-1688.

17. Haissaguerre M, Gaita F, Fischer B, Commenges D, Montserrat $P$, d'lvemois C, Lemetayer P, Warin JF. Elimination of atrioventricular nodal reentrant tachycardia using discrete slow potentials to guide application of radiofrequency energy. Circulation 1992;85:2162-2175.

18. Jackman WM, Beckman $\mathrm{KJ}$, McClelland JH, Wang $\mathrm{X}$, Friday KJ, Roman CA Moulton KP, Twidale N, Hazlitt HA, Prior MI, Oren J, Overholt ED, Lazzara R Treatment of supraventricular tachycardia due to atrioventricular nodal reentry by radiofrequency catheter ablation of slow-pathway conduction. $N$ Engl J Med 1992; 327:313-318. 